\title{
A finite volume study of the thermodynamic properties of strongly interacting matter using PNJL model.
}

\section{Subrata Sur*i}

Panihati Mahavidyalaya, Kolkata

E-mail: ssur.phys@gmail.com

\section{Abhijit Bhattacharyya \\ University of Calcutta, Kolkata \\ E-mail: abphy@caluniv.ac.in}

\section{Paramita Deb}

E-mail: paramita.deb83@gmail.com

\section{Sanjay K. Ghosh}

Bose Institute, Kolkata

E-mail: sanjayejcbose.ac. in

\section{Rajarshi Ray}

Bose Institute, Kolkata

E-mail: rajarshi@jcbose.ac.in

\begin{abstract}
We have studied the thermodynamic properties of strongly interacting matter in finite volume using Polyakov loop enhanced Nambu-Jona-Lasinio model. We considered both the 2-flavor and $2+1$ flavor matter. We observed qualitative change in the phase transition properties that resulted in the lowering of the temperature corresponding to the critical end point. Also we have calculated the susceptibilities of conserved charges for 2-flavor strongly interacting matter using PNJL model. We find substansial volume dependence for different susceptibilities.
\end{abstract}

7th International Conference on Physics and Astrophysics of Quark Gluon Plasma

1-5 February, 2015

Kolkata, India

\footnotetext{
*Speaker.

${ }^{\dagger}$ The speaker thanks the organisers of ICPAQGP 2015.
} 


\section{PNJL model in finite volume: Thermodynamics}

Experiments in heavy-ion collisions are trying to produce the quark-gluon plasma (QGP) phase of matter by colliding nuclei at ultra-relativistic energies. The matter formed due to the energy deposition of colliding particles has a finite volume. The volume of the system thus created would depend on nature of colliding neuclei, the center of mass energy and centrality of collision. Once created the system expands until it reaches freeze-out. It is important to understand the effect of finite volume on the thermodynamic properties of matter created in the experiments. For our study we use QCD inspired model of strongly interacting matter. In this work we shall use the Polyakov loop enhanced NJL (PNJL) model to study the thermodynamic properties of the strongly interacting matter in a finite volume [4]. For basic formalism of PNJL model in infinite volume the readers are referred to [1, 2, 3].We have considered PNJL model with light flavors(2 flavor) and light plus strange flavors( $2+1$ flavor). To incorporate the effect of finite volume we use a lower momentum cut-off $p_{\min }=\pi / R=\lambda$ (say), where $R$ is the system size. We start by writing down the thermodynamic potential in MFA which is given by [4]

$$
\begin{aligned}
\Omega^{\prime} & =\mathscr{U}^{\prime}[\Phi, \bar{\Phi}, T]+2 g_{S} \sum_{f=u, d, s} \sigma_{f}^{2}-\frac{g_{D}}{2} \sigma_{u} \sigma_{d} \sigma_{s}-6 \sum_{f} \int_{\lambda}^{\Lambda} \frac{d^{3} p}{(2 \pi)^{3}} E_{p_{f}} \Theta(\Lambda-|\vec{p}|) \\
& -2 \sum_{f} T \int_{\lambda}^{\infty} \frac{d^{3} p}{(2 \pi)^{3}} \ln \left[1+3\left(\Phi+\bar{\Phi} \exp \left(\frac{-\left(E_{p_{f}}-\mu_{f}\right)}{T}\right)\right) \exp \left(\frac{-\left(E_{p_{f}}-\mu_{f}\right)}{T}\right)+\exp \left(\frac{-3\left(E_{p_{f}}-\mu_{f}\right)}{T}\right)\right] \\
& -2 \sum_{f} T \int_{\lambda}^{\infty} \frac{d^{3} p}{(2 \pi)^{3}} \ln \left[1+3\left(\bar{\Phi}+\Phi \exp \left(\frac{-\left(E_{p_{f}}+\mu_{f}\right)}{T}\right)\right) \exp \left(\frac{-\left(E_{p_{f}}+\mu_{f}\right)}{T}\right)+\exp \left(\frac{-3\left(E_{p_{f}}+\mu_{f}\right)}{T}\right)\right] \\
& =\Omega-\kappa T^{4} \ln J[\Phi, \bar{\Phi}]
\end{aligned}
$$

where $E_{p_{f}}=\sqrt{p^{2}+M_{f}^{2}}$ is the single quasiparticle energy. $\Phi=\left(\operatorname{Tr}_{c} L\right) / N_{c}$ is the Polyakov loop and its conjugate is $\bar{\Phi}=\left(\operatorname{Tr}_{c} L^{\dagger}\right) / N_{c}$. In the last line $\Omega$ contains all the terms of $\Omega^{\prime}$ except the Vandermonde term. At zero baryon density we find for all the system sizes the order parameter for both $\operatorname{chiral}(\sigma)$ and deconfinement $(\Phi)$ transition smoothly passes from hadronic phase to quark phase so there is a smooth crossover. The crossover temperature is calculated and shown in table 1.

\begin{tabular}{|c|c|c|c|c|c|}
\hline & $R=2 f m$ & $R=2.5 f m$ & $R=3 f m$ & $R=5 f m$ & $R=\infty$ \\
\hline$T_{c}(\mathrm{MeV})$ (2 flavor) & 167 & 171 & 180 & 184 & 186 \\
$T_{c}(\mathrm{MeV})(2+1$ flavor) & 160 & 167 & 174 & 180 & 181 \\
\hline
\end{tabular}

Table 1: Transition temperatures for different system sizes.

From table 1 we see that $T_{c}$ has a strong dependence on system size. At non-zero quark chemical potential $\mu_{q}$ for infinite volume system the phase transition is of first-order. At some smaller $\mu_{q}$ the first order transition ends at a critical end point (CEP) where the system undergoes a second order phase transition. We have shown the phase digram as a function of system size in fig 1 . The CEP gradually shifts towards higher $\mu_{q}$ and lower $T$ and finally disappears as we reduce the volume. 

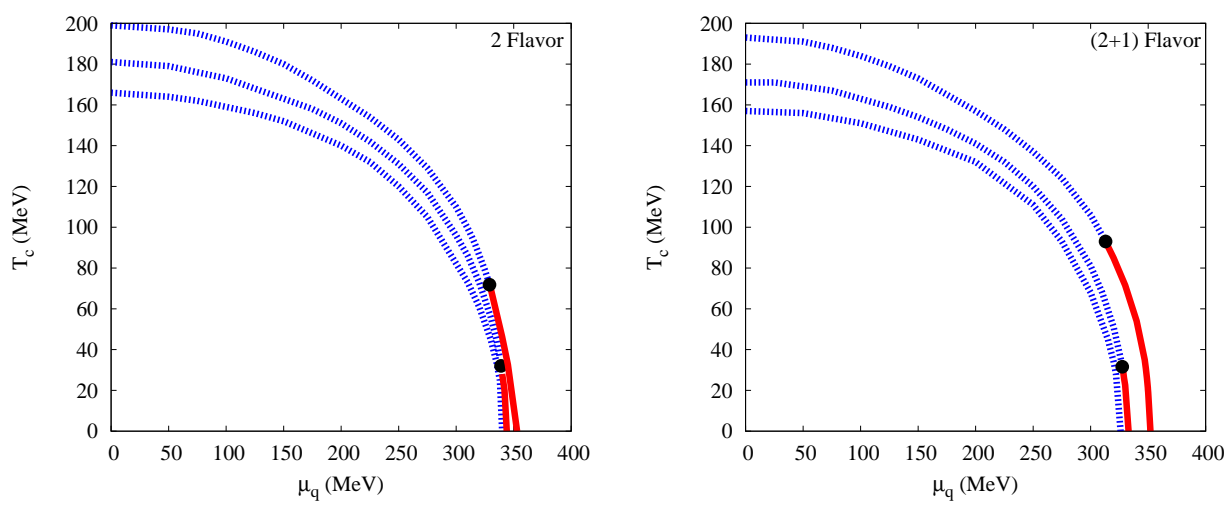

Figure 1: Phase diagram for different system sizes. The inside curve is for $R=2 \mathrm{fm}$, the next curve is for $R=2.5 \mathrm{fm}$ and the outermost curve is for $R=\infty$.
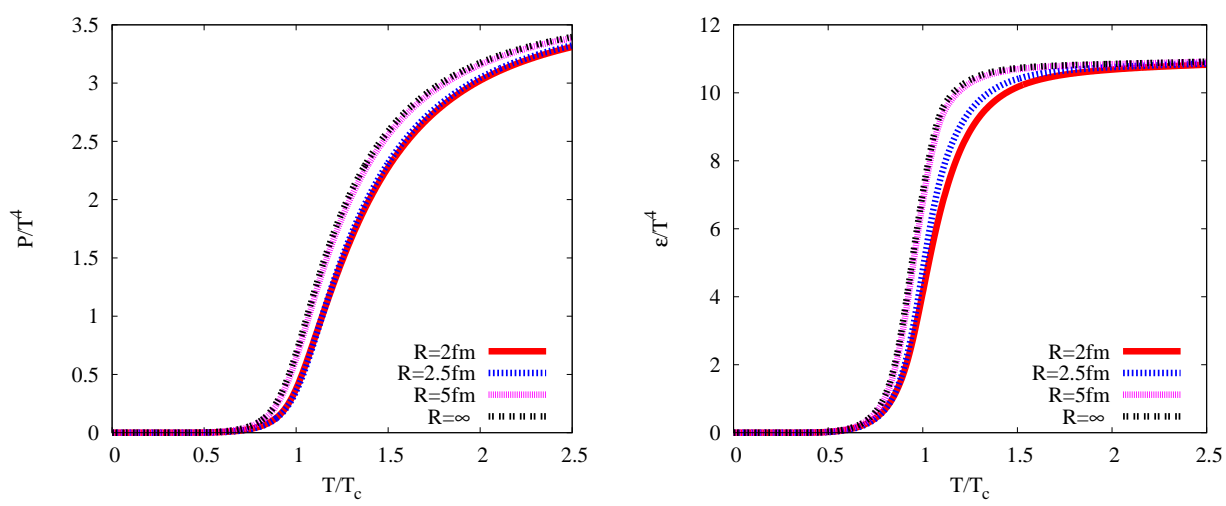

Figure 2: Pressure and energy density as a function of temperature. These plots are for 2 flavor matter only.

The pressure $P\left(T, \mu_{q}\right)=-\frac{\partial\left(\Omega\left(T, \mu_{q}\right) V\right)}{\partial V}$ and energy density $\varepsilon=-\left.T^{2} \frac{\partial(\Omega / T)}{\partial T}\right|_{V}=-\left.T \frac{\partial \Omega}{\partial T}\right|_{V}+\Omega$ both are calculated for different system sizes. In left panel of Fig. 2 we plot the temperature dependence of scaled pressure for 2 flavor system. We see significant change in scaled pressure for small system sizes. At $T_{c}$ for system size $R=2 \mathrm{fm}$ the scaled pressure is almost half of that for an infinite system. In right panel of Fig. 2 we have plotted the energy density. Here also we see significant volume dependence.

The specific heat at constant volume $C_{V}=\left.\frac{\partial \varepsilon}{\partial T}\right|_{V}$ and squared speed of sound are plotted in Fig. 3. For smaller volumes specific heat is smaller. For change in volume changes in $C_{V}$ is significant upto $T_{c}$. At large temperatures the $v_{s}^{2}$ reaches its maximum value as the system becomes almost ideal. With decreasing volume the speed of sound decreases.

\section{PNJL model in finite volume: Fluctuation}

Fluctuations are important characteristics of any physical system. Fluctuations of conserved quantum numbers are related to the respective susceptibilities via the fluctuation-dissipation theorem. The Thermodynamic aspect of phase tarnsition from hadronic phase to QGP phase can be 

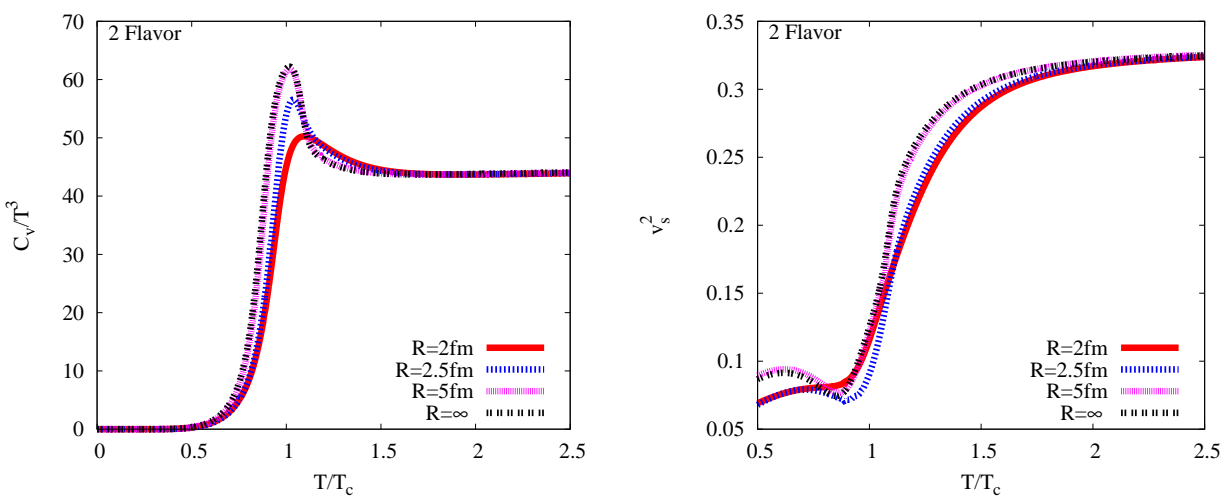

Figure 3: Variation of specific heat and squared velocity of sound with temperature for different system sizes. These plots are for 2 flavor matter only.

understood properly if we study susceptibilities of conserved charges. For 2-flavor strongly interacting system one has the quark number susceptibility(QNS) and isospin number susceptibility (INS) etc. We report here our calculation of QNS ans INS upto sixth order using 2-flavor PNJL model in finite volume [5]. Our starting point is the thermodynamical potential $\Omega$. The critical temperature is dependent on system size. We have considered different system sizes corresponding to $R=2 \mathrm{fm}, R=2.5 \mathrm{fm}, R=4 \mathrm{fm}$ and infinite volume.
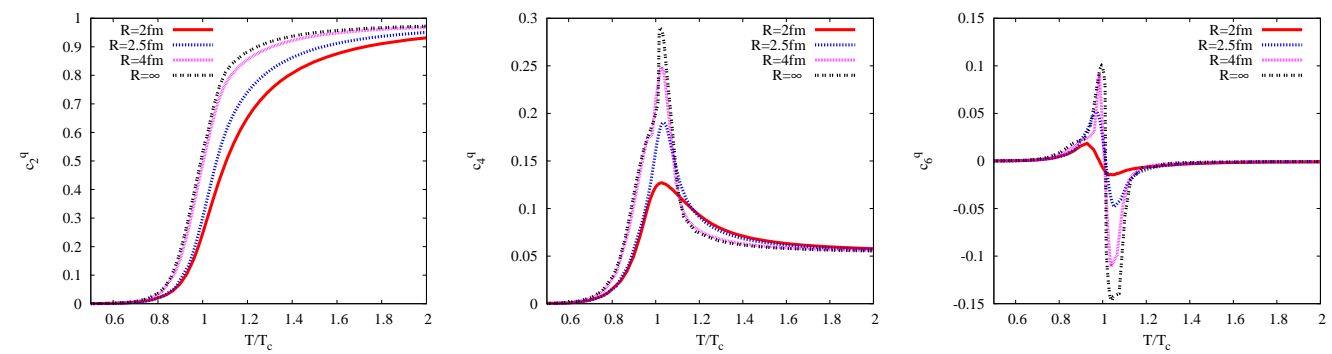

Figure 4: Variation of quark number susceptibilities with temperature for different system sizes.

Various susceptibilities of quark number and iso-spin number are defined as,

$$
c_{n}(T)=\left.\frac{1}{n !} \frac{\partial^{n}\left(\Omega\left(T, \mu_{q}, \mu_{I}\right) / T^{4}\right)}{\partial\left(\frac{\mu_{X}}{T}\right)^{n}}\right|_{\mu_{X}=0} .
$$

where $\mu_{X}=\mu_{q}$ or $\mu_{I}$ and where $T$ is the temperature and $\mu_{q}$ and $\mu_{I}$ are the quark and isospin chemical potentials respectively.

We have calculated the coefficients $c_{2}, c_{4}$ and $c_{6}$ both for quark number and isospin number susceptibilities. The variation of quark number susceptibilities with $T / T_{c}$ are shown in Fig. 4. The general features for these susceptibilities in finite volumes are quite similar to that for infinite volume. However quantitatively we observe significant volume dependence. With increase in system size there is an enhancement of all the susceptibilities. For the isospin number susceptibilities shown in Fig. 5, we find almost identical behavior. It may be noted that the most significant finite size effects are seen in the higher order susceptibilities close to the cross-over region. 

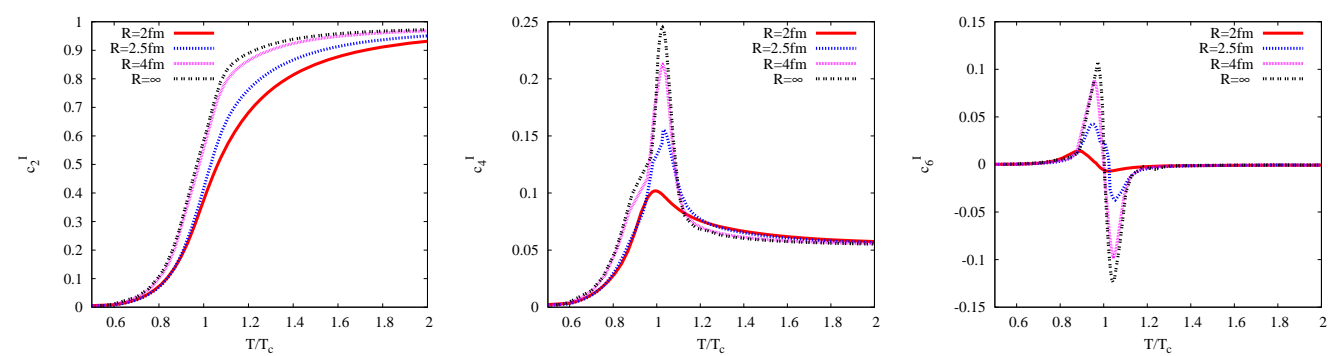

Figure 5: Variation of isospin number susceptibilities with temperature for different system sizes.

\section{Conclusion}

We have studied the thermodynamics and fluctuations of strongly interacting matter inside finite volume using PNJL model. The critical temperature $T_{c}$ has a strong dependence on system volume. With the increase of volume CEP shifts towards high $\mathrm{T}$ and low $\mu$. For $\mathrm{R}=2 \mathrm{fm}$ there is no CEP. Changes in the equation of state and speed of sound may have important consequences in the flow properties of the exotic medium created in the experiments. We find significant volume dependence in quark number and isospin number susceptibilities.

\section{Acknowledgements}

The speaker would like to thank the organiser of ICPAQGP 2015 for a stimulating conference. $\mathrm{AB}$ and RR thank DST for support.

\section{References}

[1] C. Ratti, M. A. Thaler and W. Weise, Phases of QCD: Lattice thermodynamics and a field theoretical model, Phys. Rev. D 73,014019 (2006).

[2] S. K. Ghosh, T. K. Mukherjee, M. G. Mustafa and R. Ray, Susceptibilities and speed of sound from PNJL Model, Phys. Rev. D 73, 114007 (2006).

[3] S. K. Ghosh, T. K. Mukherjee, M. G. Mustafa, R. Ray, PNJL model with a Van der Monde term, Phys. Rev. D 77, 094024 (2008).

[4] A. Bhattacharyya, P. Deb, S. K. Ghosh, R, Ray and S. Sur, Thermodynamic properties of strongly interacting matter in a finite volume using the Polyakov-Nambu-Jona-Lasinio model, Phys. Rev. D 87, 054009 (2013).

[5] A. Bhattacharyya, R. Ray and S. Sur, Fluctuation of strongly interacting matter in the Polyakov-Nambu-Jona-Lasinio model in finite volume, Phys. Rev. D 91, 051501 (2015). 